\title{
Multi-Processor based Fast Data Acquisition for a Free Electron Laser and Experiments
}

\author{
A. Agababyan, G. Asova, G. Dimitrov, G. Grygiel, B. Fominykh, O. Hensler, R. Kammering, L. \\ Petrosyan, K. Rehlich, V. Rybnikov, G. Trowitzsch, M. Winde, T. Wilksen
}

\begin{abstract}
FLASH (Free electron LASer in Hamburg) at DESY is a user facility with laser-like radiation source in the VUV and soft X-ray range. It is also a pilot facility for the future XFEL. A fast data acquisition system (DAQ) to support the accelerator operation and the user experiments was developed. The system collects data from hundreds of ADC channels in the range of $1 \mathrm{M}$ up to $2 \mathrm{G}$ samples per second. In addition it allows collecting images from digital video cameras. All the data is collected in a shared memory of a central multi-processor computer. Several processes are used to calculate e.g. the orbit, energy or photon flux, or are used as feedback loops to improve the stability of the linac. A long time archiving of the collected data from the accelerator and from the FEL experiments on a 24TB disk and finally on a tape is provided. The whole system is integrated in the DOOCS control system of FLASH. It is a novel approach to combine a fast DAQ system with a accelerator control system.
\end{abstract}

Index Terms-Accelerator control systems, Data acquisition, Free Electron Laser, Software

\section{INTRODUCTION}

$\mathrm{F}$ LASH [1] is a R\&D study exploring superconducting cavity technologies to be used by future linear accelerators and a user facility for free-electron laser experiments. During first runs the linac was operated at $0.4 \mathrm{GeV}$ to produce laser like light at a wavelength of $32 \mathrm{~nm}$. Wavelengths down to
$6 \mathrm{~nm}$ are foreseen in the near future. An electron beam of $1 \mathrm{nC}$ charge is accelerated by five cryogenic modules with eight cavities each. The bunch length is shortened by two bunch compressors to provide the required high peak currents for SASE (Self Amplified Spontaneous Emission) in the undulator section that produces the laser light. At the end of the linac the emitted light from the undulators is sent to experiments while the electron beam is deflected into a beam dump.

The same technologies will be used for the next, larger project, the XFEL, which is currently under design. The XFEL will be running at beam energies of $20 \mathrm{GeV}$ and with light wavelengths down to $0.1 \mathrm{~nm}$ hence an order of magnitude larger compared to the current implementation.

FELs require high quality diagnostics. Since the machine is operated in a pulsed mode, data of all beam-based diagnostics and the RF systems have to be recorded for every single bunch. One macro pulse can consist of up to 800 bunches. In future even 7000 bunches will be possible. With a repetition rate of 1 to $10 \mathrm{~Hz}$ data from all 1000 fast ADC channels of the diagnostics has to be recorded. This would lead to several PBytes of data per year. The described system is configurable to store for example all data of an experiment while at the same time a few channels from the accelerator could be selected for permanent storage. Additionally all data is stored on a local disk for a few weeks.

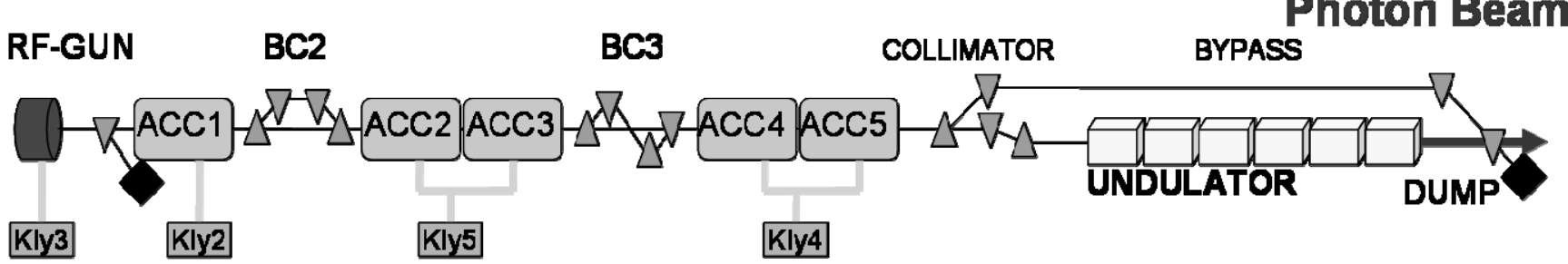

Fig. 1: The FLASH accelerator. A gun on the left side emits electrons. After acceleration in 5 modules with 8 superconducting cavities each and two bunch compressors, the photon beam is generated in the undulator stage

A. Agababyan, G. Asova, G. Dimitrov, G. Grygiel, B. Fominykh, O. Hensler, R. Kammering, L. Petrosyan, K. Rehlich, V. Rybnikov, G. Trowitzsch, M. Winde are with DESY, Hamburg and Zeuthen, Germany

T. Wilksens is with LEPP Cornell University, Ithaca, NY, USA 


\section{THE DATA ACQUISITION SYSTEM}

\section{A. Overview}

A good fraction of the diagnostics of the accelerator is read out by fast ADCs. These ADCs are sampled with the bunch frequency of $1 \mathrm{MHz}$, some with $9 \mathrm{MHz}$. The repetition rate of the accelerator is between 1 and $10 \mathrm{~Hz}$. $2 \mathrm{~ms}$ of one macro pulse of the electron beam is recorded. This time covers the duration of the beam $(800 \mu \mathrm{s})$ and the cavity filling and decay times.

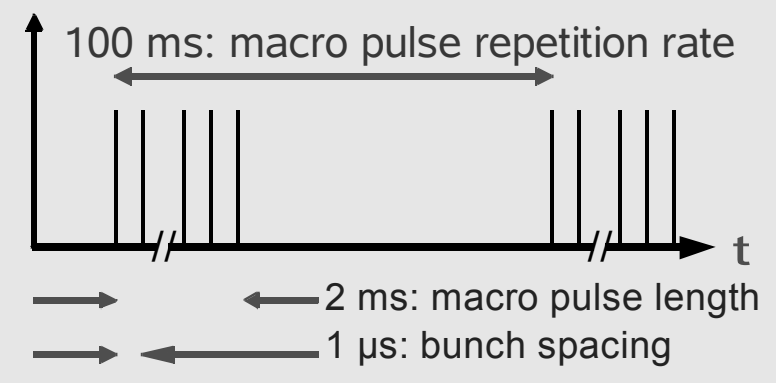

Fig. 2: The time structure of FLASH. Macro pulses have a repetition rate of 1 to $10 \mathrm{~Hz}$ with a bunch spacing of $1 \mu \mathrm{s}$

About 1000 ADC channels are distributed over tens of VME crates. To be able to analyze the ADC readings the data must be synchronized in order to correlate the same bunch of the same macro pulse from different locations of the facility.

ADC clocks and triggers are generated by a hardware timing system. The time structure of FLASH is shown in figure 2. From the timing system a stable clock is derived. This clock is in a fixed phase relation to the RF of the cavities and therefore with the beam. Trigger signals are generated by the timing to start ADCs, trigger cameras and activate the readout by the software.

In addition to the ADC data more than 30 cameras are connected to the DAQ (figure 3). These cameras vary from high pixel counts and high analog resolution types to simple cameras for observation tasks. Interfaces of these cameras are specific PCI adapters, Fire Wire networks or USB. All different camera types are handled by a common camera server to provide the same control system interface to the operators and to the DAQ system.

All other data from the control system that is required to be stored by the DAQ is collected by a so called slow collector. This collector has an interface to the control system DOOCS (Distributed Object-Oriented Control System) [2] and can by this means read all parameters of the accelerator and the experiments. Typically the readout rate of these channels is on the order of $1 \mathrm{~Hz}$. Examples are the magnet current readings.

\section{B. The Architecture}

Figure 4 shows a simplified diagram of the architecture of the implemented DAQ system. Data from the various subsystems are read by VME modules or camera servers. All the synchronization is controlled by a timing system that drives the clocks of the ADCs and the triggering of the hardware and the software. An event every 16 machine macro pulses, distributed via the timing system, is used to mark the data blocks with a unique identifier in all subsystems. An interrupt at the end of an accelerator macro pulse is used to start the readout. After processing, the data is sent to collector processes. It is the task of these collectors to ask for retransmission of lost data blocks and to sort them into the central shared memory. Various middle layer services are attached to this shared memory. These services are used for measurements, calibrations, or feedback loops. The results can be sent back to the shared memory, displayed to users or used to steer actuators in the accelerator. The distributor is a further consumer of the data in the shared memory. It picks out events and channels and sends the data to an event builder. The event builder finally writes the data into files in separated streams for e.g. the FEL experiments and the accelerator.

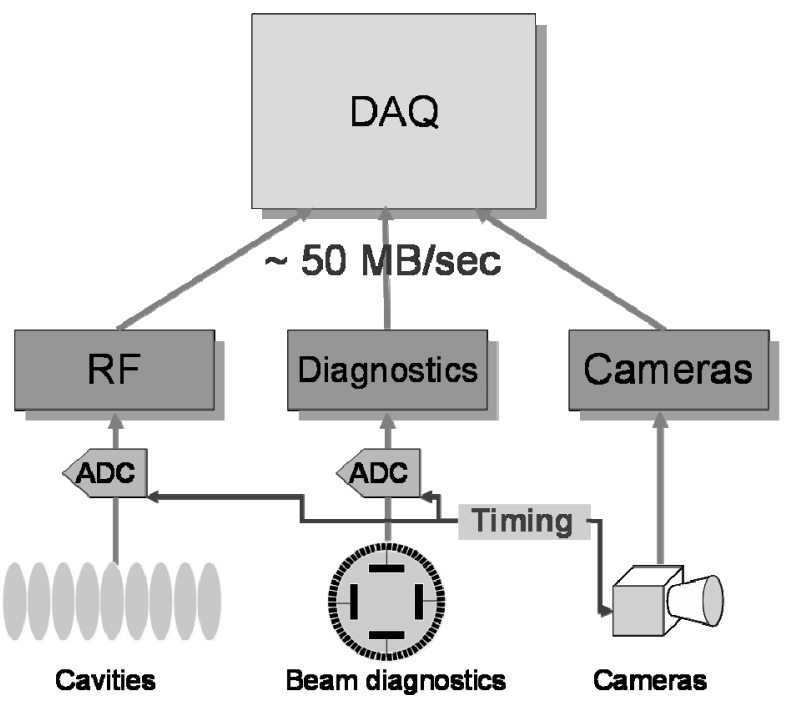

Fig. 3: Data from all distributed devices are synchronized by the timing system and send to the DAQ

A feature of the developed architecture is that it can run multiple instances of the DAQ in parallel. The front-end servers send their data as Multicast packets to the network. No additional load is introduced on the front-ends if further DAQs participate in the data flow. This is important since usually the front-ends have less powerful CPUs.

\section{The Front-end}

Most of the fast Hardware is connected to modules in VME crates controlled by CPU boards running Solaris. The cameras are connected to PCs with a LINUX operating system. All hardware is interfaced to the control system by DOOCS device server processes. The DOOCS interface provides full access to the data and configuration parameters. Local archiving of measured values or results from calculations is part of the standard server library. Waveforms read by the ADCs are therefore also provided on the network. The server keeps the waveforms of the most recent 16 accelerator macro pulses available to the control system. The DAQ described in 
this paper goes beyond this storage of single values per channel and allows a permanent storage of complete waveforms.

For the adoption to the DAQ system the front-end server reads the data from VME by DMA into the CPU memory after an interrupt from the timing system. The data is then converted and corrected to real physical units and copied to an output buffer. It is the task of a sender library to transfer the buffer to the network by a Multicast UDP protocol.

\section{Data Collectors}

The packets sent by the front-ends are received by collector processes. In case of losses the collector has to ask a front-end for retransmission of lost packets. Normally there is no handshake between the collector and the device server. Measurements show that about one percent of the packets have to be retransmitted only. This was caused by an older network switch. After the upgrade of the central network equipment the loss rate was reduced significantly. The links to the front-ends are handled in independent threads. After receiving packets the data is sorted by time or event number into the correct position of the shared memory.

Different types of collectors have been developed. One is used for the fast ADC channels, another one is for images from camera servers, and a further one is used for all so called slow channels. Slow channels are typically collected with a rate of $1 \mathrm{~Hz}$ and are used for single values like magnet current readings.

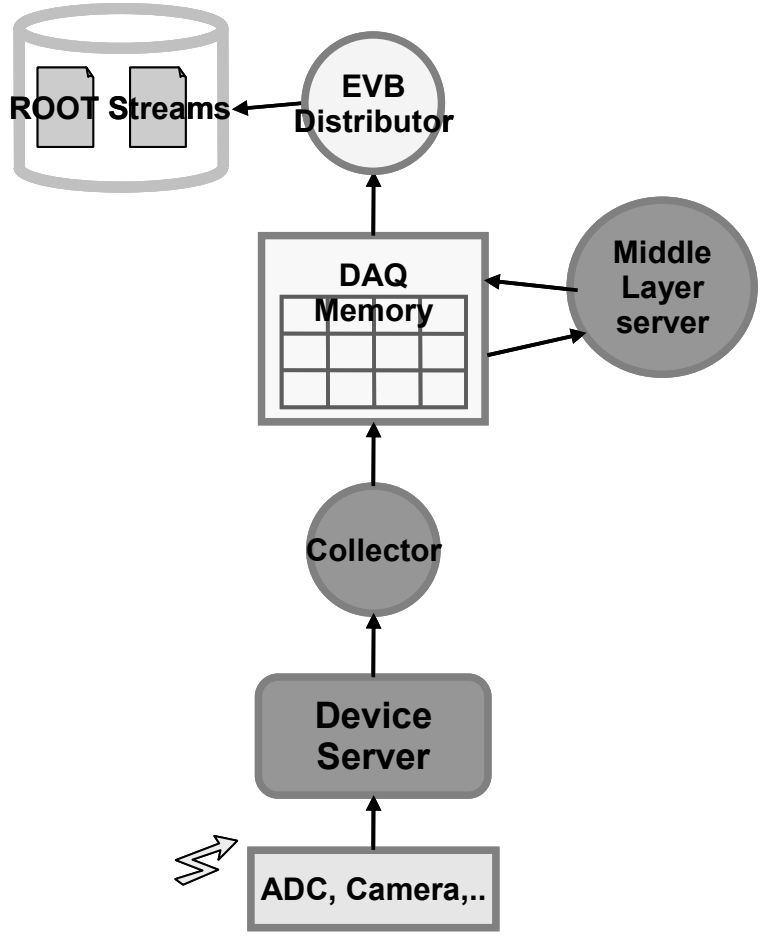

Fig. 4: Overview of the architecture of the DAQ system

A typical run of FLASH generates up to $50 \mathrm{MB}$ per second from all collectors. Although the hardware is already two year old, a factor of two higher data rate should be no problem.

\section{E. Buffer Manager}

The core of the DAQ system is the buffer manager. It coordinates all read and write operations to the shared memory. All processes with interest in getting or setting data from/to the shared memory have to use readers and writers of the buffer manager. The buffer manager library takes subscription to data channels and delivers the requested data to subscribers when all buffers are available from one accelerator macro pulse.

All processes that are attached to the shared memory implement the DOOCS server library. Therefore they can be managed by standard control system calls and tools. This includes the finite state machines built into all processes. Configuration parameters are sent as XML structures to all parts of the DAQ. Again, the DOOCS protocol is used.

The hardware for the central DAQ is a Sun Server E2900 with 32 GB of memory shared among 8 dual core CPUs. The architecture of this server provides a very high bandwidth to the shared memory for all processors. Since the front-ends send the data as Multicast packets, several central DAQ systems can run in parallel. Configurations with one to three independent DAQs on one computer or on several computers have been successfully tested.

\section{F. Middle Layer Processes}

The central shared memory contains all beam relevant information. This makes it an ideal place for attaching measurement, calibration and even slow feedback processes. The electron energy measurement in a dogleg of FLASH is a good example. The calculation requires all bunch information of three beam position monitors and a few magnet readings. The algorithm is implemented in MATLAB. To ship the data into and out of MATLAB a framework was developed. The library provides input, filter and output channels. All channels are online configurable to select data from the shared memory, filter data and input a structure to a MATLAB procedure. This procedure creates the results in a structure that is then passed to the output channels. The output channels can be directed to a further procedure, back to the shared memory or sent to any channel of the control system.

With this schema an implementation of slow feedbacks is straight forward. Adding further control loops does not create any further load on the front-ends since the data is usually available in the shared memory. If the processors of the central DAQ server run out of free CPU cycles, a further CPU box can be added, again without increasing the front-end load. This makes the system quite scaleable.

The framework provides some debugging features. Since all input and output channels can be configured via the network, it is possible to switch off or redirect the outputs and inputs. All channels provide archiving of the values. This can be used to monitor intermediate calculations or status bits of the control algorithm. 


\section{G. Distributor and Event Builder}

A distributor process reads the data from the shared memory to store them on disk and tape. This process is configured by the run controller to pick out certain events and channels for each of its streams. A stream is a collection of channels that are written into separate ROOT [3] files. Typically one stream is set up for the accelerator, a further stream for an experiment of the accelerator and a third one for a FEL experiment. Often the experiments are handled in a separate DAQ system running in parallel to decouple the different run periods of the experiments.

The event builder receives the streams from the distributor via a TCP/IP link. It therefore can sit on a different computer. It sends the data to writer processes. Writers can produce different output formats. The FLASH DAQ produces ROOT files only so far. Writing of ROOT files is slower than the event builder input rate because of the compression. To cope and is normally compressed. The compression gives a factor of three reduction of the FLASH data. A careful design of the ROOT file layout is required since the time to retrieve data from the files depends strongly on it.

The main DAQ server has a small amount of disk space (2TB). Copy jobs are used to write the ROOT files to dCache [4] and to a local disk server. dCache is a hierarchical disk and tape storage system developed for the HEP (High Energy Physics) experiments at DESY and Fermilab. For FLASH it is mainly used as a long-time archive for the user experiments. The local disk server provides 24TB of storage in a Zeta Byte File system (ZFS) [5]. It is based on a Sun X4500 server with 2 dual-core $2.6 \mathrm{GHz}$ AMD processors. 20TB of space are needed to keep the accelerator data of about 30 days. Most of the linac data is not required to be saved on tape. Nevertheless, a month is enough to analyze certain events or to decide to save the data on dCache.

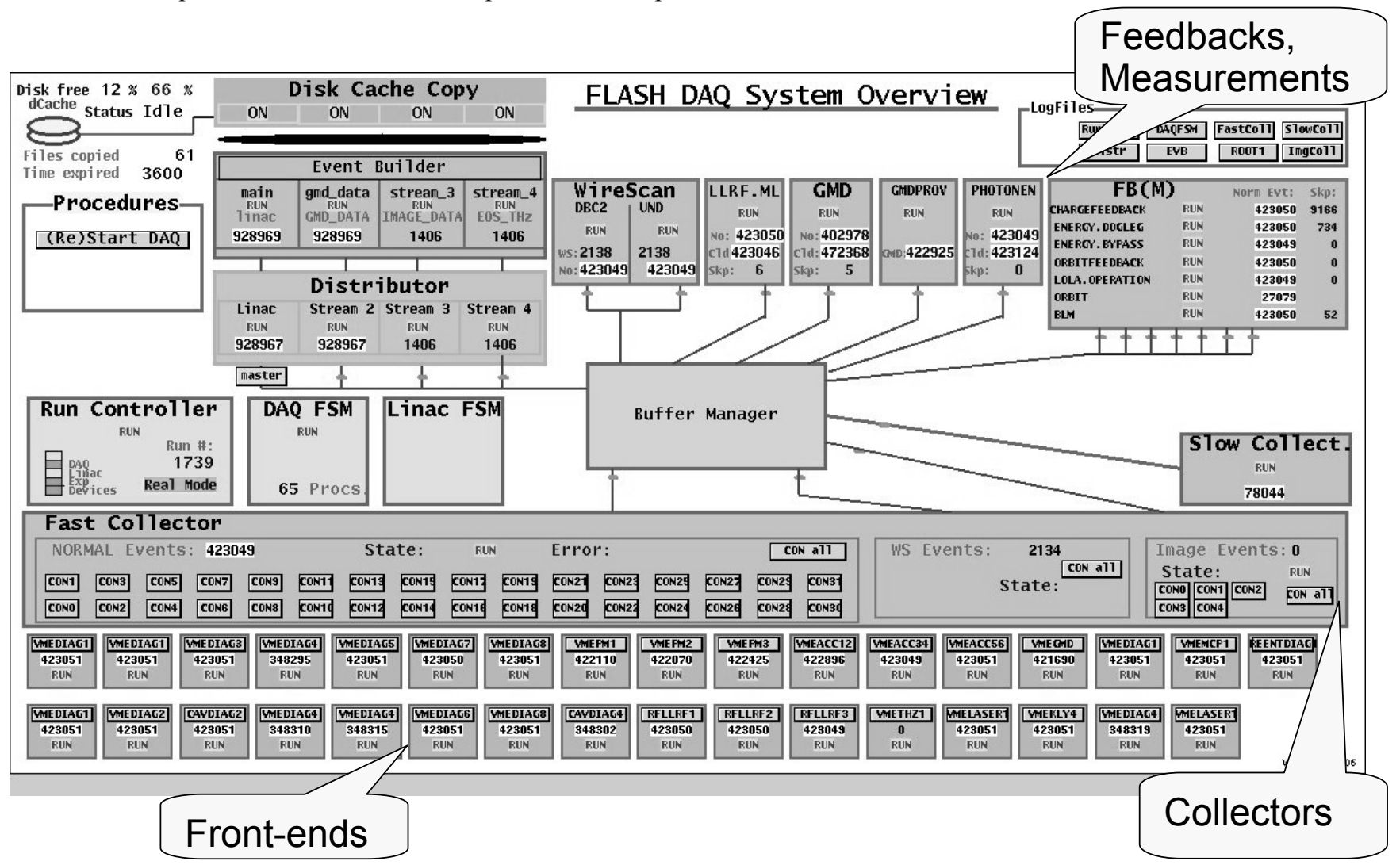

Fig. 5: DOOCS online display of the running DAQ processes

with the data rates, several parallel running processes writing parallel files are used. The event builder can automatically adapt to varying data rates by connecting to additional writer processes.

\section{H. Data Storage}

The ROOT system was developed at CERN for High Energy Physics experiments. It is a self-describing data format with a lot of supporting tools. Data is internally stored in trees

\section{Run Controller}

The whole DAQ system is distributed on 100 processes. It is the task of the Run Controller to configure all processes and to bring them into the right state. Since the accelerator and the experiments can run in different modes, the environment demands a configurable system. Channel names to be passed to the DAQ system, amount of data per channel, event types and streams to be written are examples of the parameters that 


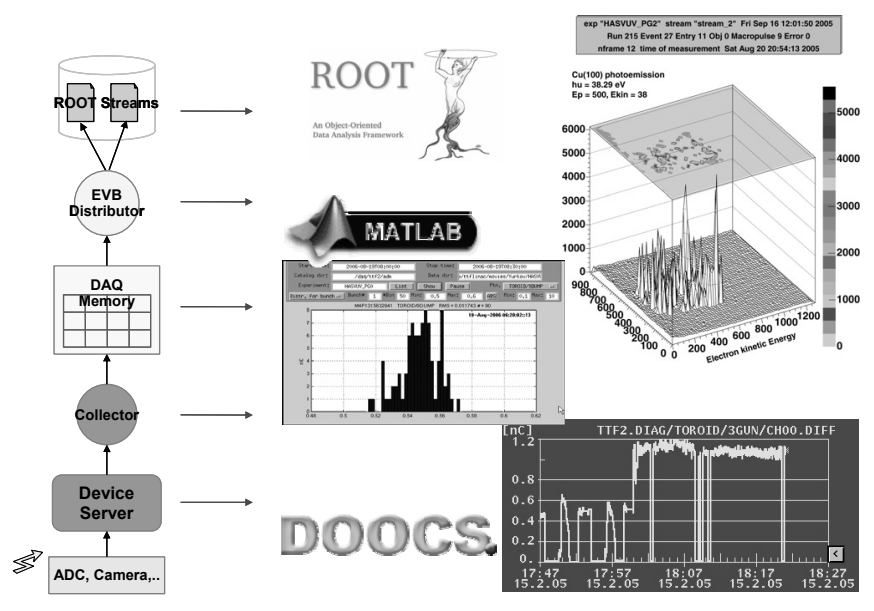

Fig. 6: DOOCS, MATLAB and ROOT tools are used to display values

are stored in a ORACLE data base. Java programs provide the configuration interface for administrators and experiments. During a start of a run the configuration data is downloaded to all contributing processes as XML strings or numerical values.

All processes implement a Finite State Machine (FSM) to keep all subsystems synchronized. The FSM steps through 'initialized', 'configured', 'ready', 'prepared', 'run' and 'paused' according the commands of the run controller. A central FSM observes all processes in the DAQ system and keeps them running.

\section{J. Control System Integration}

DOOCS is the control system of FLASH. It integrates all subsystems of the accelerator and many devices of the FEL experiments. Therefore it was advisable to integrate the DAQ system components as well. Since all DAQ processes implement the DOOCS server library the tools of the control system have access to the DAQ parameters. Figure 5 shows the DAQ status overview. This panel was created by the standard DOOCS graphical editor program $d d d$.

Furthermore, an interface to the ROOT files was developed to allow programs written in MATLAB to access the stored data in addition to all other values in the control system. It is planned for the future to create an interface to the ROOT files with standard API calls of DOOCS. This would allow displaying actual graphs with archived data from the DAQ system in the same plot.

\section{OPERATIONAL EXPERIENCE}

In summer 2005 the first FEL experiments came into operation. At this time the core part of the DAQ was ready as well and the data of the ADCs and cameras were stored in ROOT files. The analysis of the data was performed by ROOT routines. In 2006 the first middle layer servers went into standard operation. A few bugs had to be corrected and patches for the operating system were required. Afterwards the system ran very stable. More middle layer processes were added and the described framework to integrate MATLAB procedures was brought into operation.

1.7TB of data was stored on tape in $2005,3.7$ TB in 2006 and $1.2 \mathrm{~TB}$ in the first three month of the run period 2007. During the first year of operation we found the architecture very helpful in implementing slow feedback processes. In the future it is planned to implement even more control loops to stabilize further parameters of the accelerator. Improvements on universal user interfaces are still required. A simpler access for the operators to the stored data is under development.

\section{CONCLUSION}

The described DAQ system for FLASH is a novel combination and integration of an accelerator control system with the techniques of a HEP DAQ system. It was shown that an integrated framework can solve the task to collect and control slow data and devices, complex beam instrumentation and equipment with high data rates.

More than a year of routine operation has proven that the overall architecture and design is capable to support and sustain the reliable operation of such a complex machine like the FLASH. Furthermore the chosen design did show the scalability needed for the upcoming generation of FEL facilities.

\section{REFERENCES}

[1] Rossbach et al, "Generation of GW radiation pulses from a VUV freeelectron laser operating in the femtosecond regime", Phys. Rev. Lett. 88, 104802 (2002).

[2] K. Rehlich et al, "DOOCS: an Object Oriented Control System as the integrating part for the TTF Linac", Proceedings ICALEPCS '97, Beijing, China. Available: doocs.desy.de

[3] R. Brun and F. Rademakers, "ROOT - An Object Oriented Data Analysis Framework", Proceedings of the AIHENP'96 Workshop, Lausanne, Sep. 1996, Nucl. Inst. \& Meth. in Phys. Res. A 389 (1997) 81-86. Available: root.cern.ch

[4] P. Fuhrmann et al, "dCache, a distributed Storage data caching system", Proceedings of CHEP 2001, Beijing, China., Available: dcache.desy.de

[5] Sun Microsystems, Inc., "ZFS (Zettabyte File System)", Available: www.opensolaris.org/os/community/zfs 